\title{
MULTIPLE SOLUTIONS AND PERIODIC OSCILLATIONS IN NONLINEAR DIFFUSION PROCESSES*
}

\author{
DONALD S. COHEN†
}

\begin{abstract}
We study the oscillatory stationary states in the temperature and concentration fields occurring in tubular chemical reactors. Singular perturbation and multitime scale procedures are combined formally to clearly and simply reveal the mechanism controlling these oscillatory states. Their stability is also studied, and when coupled with previously obtained results on multiple steady states, this information completes the response (bifurcation) diagram in one-parameter range of the tubular reactor. The results apply also to more general nonlinear parabolic problems of which the first order tubular reactor is a special case.
\end{abstract}

1. Introduction. One of the more interesting recent developments in chemical reactor theory has been the discovery of oscillatory stationary states in the temperature and concentration fields. For the most part these have been observed experimentally [1] or by some machine computation [1]-[3]. In the case of a simple stirred tank reactor, Hlavacek, Kubicek and Jelinek [4] have given analytical plausibility arguments for the appearance of limit cycles. However, there seems to be no treatment of the more difficult nonadiabatic tubular flow reactors. Such a study is given here.

The entire question of oscillatory solutions and their stability is intimately connected with previous considerations of bifurcation, multiplicity, existence, and stability of positive solutions of certain parabolic initial-boundary value problems describing the tubular reactors. These are examined in depth by A. B. Poore [5], [6] who has studied all these questions for the system

$$
\begin{aligned}
& T_{t}=\frac{1}{P} T_{x x}-T_{x}-\kappa\left(T-T_{c}\right)+D B(1-C) e^{T /(1+\gamma T),} \\
& 0<x<1, \quad t>0, \\
& C_{t}=\frac{1}{P} C_{x x}-C_{x}+D(1-C) e^{T /(1+\gamma T)}, \\
& 0<x<1, \quad t>0,
\end{aligned}
$$

$$
\begin{aligned}
T_{x}(0, t)-h T(0, t) & =0, \quad t \geqq 0, \\
C_{x}(0, t)-l C(0, t) & =0, \quad t \geqq 0, \\
T_{x}(1, t) & =0, \quad t \geqq 0, \\
C_{x}(1, t) & =0, \quad t \geqq 0, \\
T(x, 0) & =\Phi(x), \quad 0 \leqq x \leqq 1, \\
C(x, 0) & =\Psi(x), \quad 0 \leqq x \leqq 1 .
\end{aligned}
$$

* Received by the editors June 20, 1972, and in revised form December 7, 1972.

† Department of Applied Mathematics, California Institute of Technology, Pasadena, California 91109. This work was supported in part by the U.S. Army Research Office (Durham) under Contract DAHC-04-68-C-0006 and by the National Science Foundation under Grant GP 18471. 
These are the equations for the temperature $T$ and concentration $C$ in a nonadiabatic tubular reactor, and the other quantites are prescribed physical constants.

By appropriate simple transformations, the question of oscillatory solutions can be reduced to a study of

$$
\begin{aligned}
u_{t}=\frac{1}{\varepsilon} u_{x x}-u_{x}+[\alpha(\lambda)+1] u-\beta(\lambda) w & +f(\lambda, u, w)], \\
\quad 0<x<1, \quad t>0, & \\
w_{t}=\frac{1}{\varepsilon} w_{x x}-w_{x}+\beta(\lambda) u+[\alpha(\lambda)+1] w+g(\lambda, u, w), & \quad 0<x<1, \quad t>0, \\
u_{x}(0, t)-\varepsilon u(0, t) & =0, \quad t \geqq 0, \\
w_{x}(0, t)-\varepsilon w(0, t) & =0, \quad t \geqq 0, \\
u_{x}(1, t) & =0, \quad t \geqq 0, \\
w_{x}(1, t) & =0, \quad t \geqq 0, \\
u(x, 0) & =\phi(x), \quad 0 \leqq x \leqq 1, \\
w(x, 0) & =\psi(x), \quad 0 \leqq x \leqq 1 .
\end{aligned}
$$

The motivation for obtaining this form of the equations is given in [5] and is common in the chemical engineering literature [3], [4]. Here $u$ and $w$ respectively represent the nondimensionalized temperature and concentration of equations (1.1)-(1.8). The other quantities are known from the physical situation, and we shall state their properties in $\S 2$.

We shall study the system (1.9)-(1.16) in the case that the Peclet number $\varepsilon$ is small; i.e., $0<\varepsilon \ll 1$. In $\S 2$ we briefly summarize the pertinent previously developed theory for the system (1.9)-(1.16), and we shall collect certain mathematical machinery which we shall need in the subsequent analysis of this problem. In $\S 3$ by a simple singular perturbation procedure we show that to first order in $\varepsilon$ the study of (1.9)-(1.16) can be reduced to the consideration of a far more tractable set of nonlinear ordinary differential equations. In $\S 4$ we analyze the nonlinear ordinary differential equations specifically for oscillatory solutions. This is accomplished formally by a multitime scale method (i.e., the so-called "two-timing" method [7]). Not only does this method produce the periodic solutions, but the stability of the solutions is also immediately resolved without recourse to further techniques. In $\S 5$ we indicate a more complex situation which occurs in the system (1.9)-(1.16) for chemical processes more complicated than those studied in $\S \S 2$ to 4 .

2. Summary of previous results and heuristic motivation for new results. In order to establish the results of the present paper, we shall need certain previously established facts concerning the steady state problem corresponding to (1.1)-(1.8). Rather than rederive these results we shall simply state them and refer the reader to the appropriate mathematical and chemical engineering literature. 


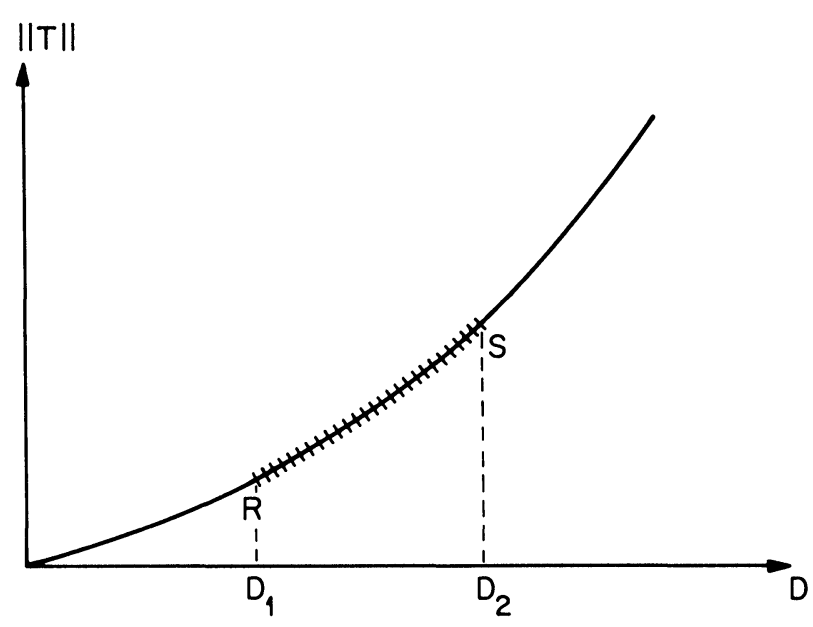

FIG. 1

It is well established [8]-[11] that there can exist multiple steady (i.e., $\partial / \partial t \equiv 0$ ) states for the system (1.1)-(1.8). In fact, for all ranges of the physical parameters the numbers of these steady states and their (infinitesimal linearized) stability is known [5], [11]. Two distinct situations will concern us here. These are best described by referring to Figs. 1 and 2. In both figures we illustrate the behavior of the amplitude of the temperature $\left(\|T\|=\max _{0 \leqq x \leqq 1}[T(x)]\right)$ as the parameter $D$ (the Damkohler number) varies. Such figures are called response diagrams.

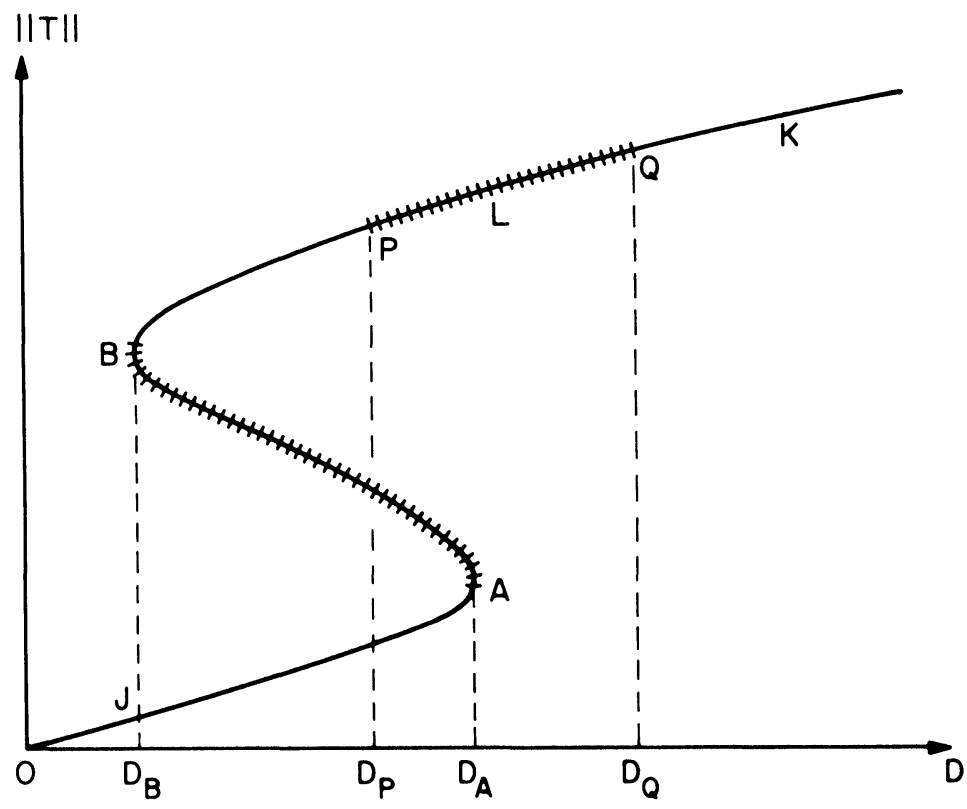

FIG. 2 
Figure 1 shows that for values of the physical parameters $\kappa, B, P, T_{c}, \gamma, h, l$ in certain ranges (and these ranges are given explicitly by A. B. Poore [6]), there is a unique solution of the steady state problem for all values of the Damkohler number $D$. The cross-hatching represents instability; that is, based on linearized stability theory for the system (1.1)-(1.8) it is found that for all values of $D$ in $D_{1} \leqq D \leqq D_{2}$ the unique steady state is unstable. Based on both experiments and machine computation for special values of the parameters, R. A. Schmitz [2] has concluded that in such situations an oscillatory stationary state is set up, so that, in fact, no stable steady state does exist, but a periodic solution of the time-dependent equations (1.1)-(1.8) evolves for $D$ in the range $D_{1} \leqq D \leqq D_{2}$. Thus, it is reasonable to expect that points $R$ and $S$ are bifurcation points for the time-dependent (parabolic) problem (1.1)-(1.8), and from these points branches of periodic solutions bifurcate. In $\S 4$ we shall show (formally) that this is indeed the case.

Figure 2 illustrates a steady state response diagram for a different set of values of the parameters [6]. The cross-hatching again represents instability. In this case we shall establish (also formally) that the points $P$ and $Q$ are bifurcation points for the time-dependent problem, and from these points branches of periodic solutions bifurcate.

Our illustrations, Figs. 1 and 2, indicate two of the six distinct situations which occur for the steady state problem corresponding to equations (1.1)-(1.8). All six cases and the conditions for their occurrence are given by A. B. Poole [5]. We have described two typical situations here. Our analysis applies equally well to all cases, and it completely resolves the stability considerations of the response diagrams for the nonadiabatic tubular reactor. Thus, for example, Fig. 1 indicates that in the steady state as $D$ is increased, so also is $\|T\|$ increased and a new unique steady state is set up until $D$ reaches $D_{1}$. Our results $(\S 4)$ then imply that a small amplitude oscillation (a stable stationary state) is then set up. As $D$ is further increased, it appears that this oscillatory state first increases in amplitude and then decreases in amplitude until the oscillation vanishes at $D=D_{2}$. As $D$ is further increased, the solution again becomes the unique stable steady state solution.

The stability story is more complex for the response diagram of Fig. 2. We shall trace the process as $D$ is increased starting at a small amplitude stable steady state for $D$ near $O$. The response moves along the path $O J A$. As $D$ is increased past $D_{A}$, the temperature undergoes a jump to a large amplitude stable periodic response above point $L$, and as $D$ is further increased, the amplitude of this oscillation decreases until the oscillation vanishes at point $Q$. The stable steady state is followed up the branch through point $K$ as $D$ increases still further. Now, as $D$ is decreased, the process follows the branch $K Q L P B$, the solution being steady from $K$ to $Q$ and from $P$ to $B$ with a stable oscillation of increasing then decreasing amplitude from $Q$ to $P$. As $D$ is decreased below $D_{B}$, there is an extinction as the response jumps to point $J$ and then follows the path $J$ to $O$.

The above arguments are plausible and indeed very satisfying within the context of chemical reactor theory. We shall establish their truth in the next sections. (However, under conditions other than those considered here other types of phenomena are possible, and we discuss these possibilities in $\S 5$.) The main points are that for the time-dependent nonlinear equations governing nonadiabatic tubular reactors, the points $R$ and $S$ of Fig. 1 and $P$ and $Q$ of Fig. 2 are bifurcation 
points and that from these bifurcation points at least two distinct solution branches emanate. One branch represents a solution not depending explicitly on time (but neverthless still a solution of the time-dependent equations), and the other branch at each bifurcation point represents a branch of time-periodic solutions. Thus, the bifurcation diagrams for the time-dependent system look like those illustrated in Figs. 3 and 4.

C. R. McGowin and D. D. Perlmutter [11] and A. B. Poore [6] have given complete tabulations of all the points on the steady state response diagrams for (1.1)-(1.8) where changes of (linearized) stability take place. (Poore gives complete analytical characterizations of these points for small $\varepsilon>0$.) Thus, the values and characterizations of $T, C$, and $D$ at the points $R, S, P, Q$ of Figs. 1 and 2 are known. The present analysis begins at this stage. Suppose, for example, that $T_{1}(x)$ and $C_{1}(x)$ represent the steady state solutions at point $P$ of Fig. 2 when $D=D_{P}$. This corresponds to the solution $u \equiv w \equiv 0$ at $\lambda=\lambda_{0}$ of (1.9)-(1.16). Hence, $u \equiv w \equiv 0$ at $\lambda=\lambda_{0}$ are solutions corresponding to the steady state solutions of (1.1)-(1.8), and we shall look for the bifurcation of time-periodic solution branches from this point. In fact, equations (1.1) $-(1.8)$ take the form (1.9) $-(1.16)$ near any of the points $R, S, P$, or $Q$. Therefore, our arguments of this section will be established by a demonstration that stable oscillatory solutions bifurcate from the point $\lambda=\lambda_{0},\|u\|=\|w\|=0$.

Motivated by all the preceding discussion, we now study the system(1.9)-(1.16) under the following conditions:

H-1: $f(\lambda, u, w)$ and $g(\lambda, u, w)$ are smooth functions of $(\lambda, u, w)$ satisfying $f\left(\lambda_{0}, 0,0\right)=g\left(\lambda_{0}, 0,0\right)=0$. that is,

H-2: $f$ and $g$ contain no linear terms in $u$ and $w$ near $(\lambda, u, w)=\left(\lambda_{0}, 0,0\right)$;

$$
\begin{gathered}
f\left(\lambda_{0}, 0,0\right)=f_{u}\left(\lambda_{0}, 0,0\right)=f_{w}\left(\lambda_{0}, 0,0\right)=0 \\
g\left(\lambda_{0}, 0,0\right)=g_{u}\left(\lambda_{0}, 0,0\right)=g_{w}\left(\lambda_{0}, 0,0\right)=0
\end{gathered}
$$

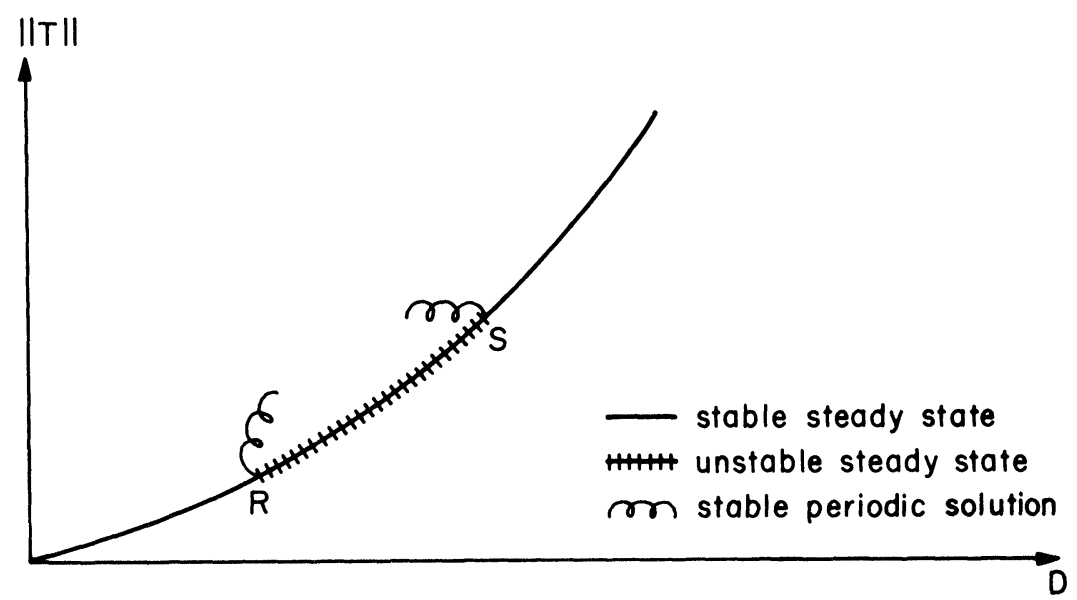

FIG. 3 


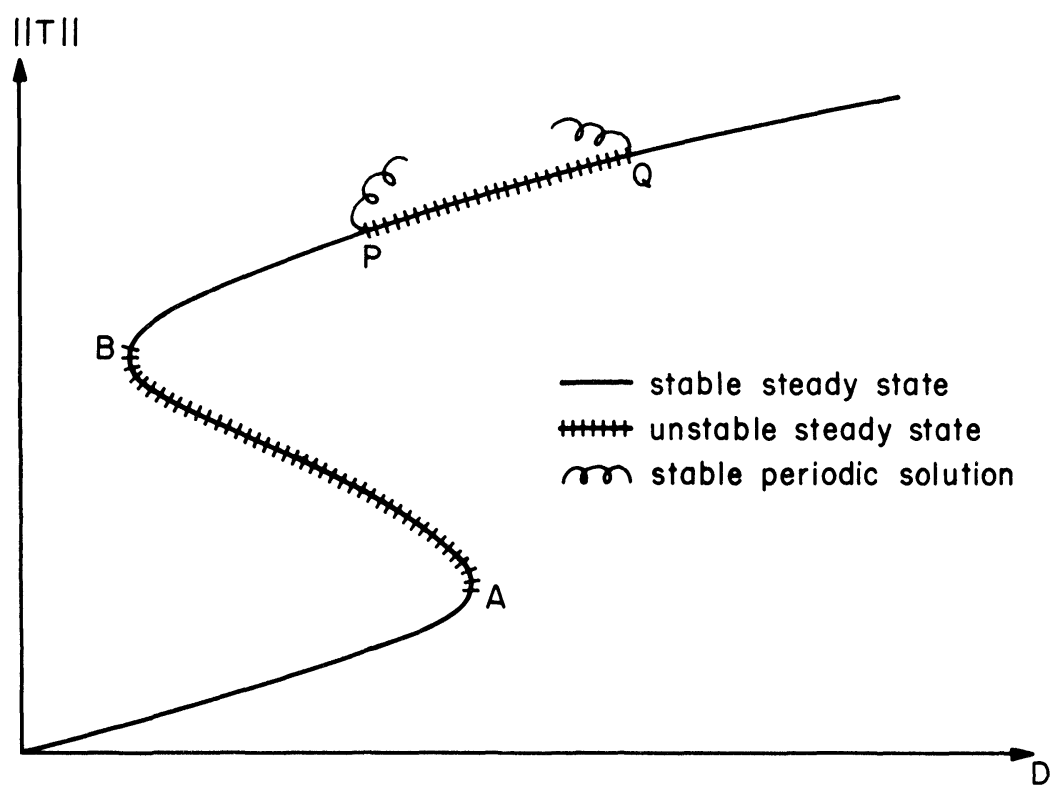

FIG. 4

H-3: $\alpha(\lambda)$ and $\beta(\lambda)$ are smooth functions of $\lambda$ satisfying $\alpha\left(\lambda_{0}\right)=0$ for some $\lambda=\lambda_{0}$, and $\beta(\lambda)>0$ for all $\lambda$.

H-4 : $\varepsilon>0$ satisfies $0<\varepsilon \ll 1$, and all other quantities are $O(1)$.

Within the framework of the nonlinear diffusion processes motivating our study, the functions $f, g, \alpha$ and $\beta$ are, in fact, infinitely continuously differentiable functions of all their arguments. Strictly speaking, we do not need such strong smoothness requirements for our analysis; however, for ease of presenting the main ideas in this paper we shall assume that "smooth" implies this strong requirement of $C^{\infty}$-functions. The hypothesis $\mathrm{H}-2$ is simply a formulation of the fact that in performing the transformations to take (1.1)-(1.8) into (1.9)-(1.16), we have explicitly subtracted the linear terms near $(\lambda, u, w)=\left(\lambda_{0}, 0,0\right)$ from the nonlinearities. Thus, the linearized system about $(\lambda, u, w)=\left(\lambda_{0}, 0,0\right)$ is simply equations (1.9) $-(1.16)$ with the functions $f$ and $g$ deleted. The value $\lambda=\lambda_{0}$ corresponds to the value of $D$ at the points $R, S, P$, or $Q$ of Figs. 1 and 2. At such a point it is found that $\alpha\left(\lambda_{0}\right)=0$. Thus, our problem (1.9)-(1.16) under the conditions $\mathrm{H}-1$ to $\mathrm{H}-4$ contains the problem of the nonadiabatic tubular reactor as a special case and encompasses a much wider class of problems.

3. The reduced system. By a singular perturbation procedure we shall now reduce the problem (1.9)-(1.16) to a somewhat simpler system of nonlinear ordinary differential equations which we shall study in $\S 4$ using the "two-timing" method.

Assume that $0<\varepsilon \ll 1$. We now construct the asymptotic expansions of the solutions of (1.9)-(1.16) as $\varepsilon \rightarrow 0$. In this case we find by the techniques of singular perturbation theory [7] that there is an initial boundary layer of thickness $O(\varepsilon)$ near $t=0$ for all $x$ in $0 \leqq x \leqq 1$. Away from this boundary layer the form of the 
asymptotic expansion (the outer solution) is given by

$$
u(x, t, \varepsilon) \sim \sum_{n=0}^{\infty} u_{n}(x, t) \varepsilon^{n}, \quad w(x, t, \varepsilon) \sim \sum_{n=0}^{\infty} w_{n}(x, t) \varepsilon^{n} .
$$

Inserting (3.1) into equations (1.9)-(1.14), we find by equating like powers of $\varepsilon$ that to order $\varepsilon^{2}$ we obtain

$$
\frac{\partial^{2} u_{0}}{\partial x^{2}}=0, \quad \frac{\partial^{2} w_{0}}{\partial x^{2}}=0
$$

$$
\begin{gathered}
\frac{\partial u_{0}}{\partial x}(0, t)=0, \quad \frac{\partial w_{0}}{\partial x}(0, t)=0 \\
\frac{\partial u_{0}}{\partial x}(1, t)=0, \quad \frac{\partial w_{0}}{\partial x}(1, t)=0 \\
\frac{\partial^{2} u_{1}}{\partial x^{2}}=\frac{\partial u_{0}}{\partial t}+\frac{\partial u_{0}}{\partial x}-[\alpha(\lambda)+1] u_{0}+\beta(\lambda) w_{0}-f\left(\lambda, u_{0}, w_{0}\right) \\
\frac{\partial^{2} w_{1}}{\partial x^{2}}=\frac{\partial w_{0}}{\partial t}+\frac{\partial w_{0}}{\partial x}-\beta(\lambda) u_{0}-[\alpha(\lambda)+1] w_{0}-g\left(\lambda, u_{0}, w_{0}\right) \\
\frac{\partial u_{1}}{\partial x}(0, t)=u_{0}(0, t) \\
\frac{\partial w_{1}}{\partial x}(0, t)=w_{0}(0, t) \\
\frac{\partial u_{1}}{\partial x}(1, t)=0 \\
\frac{\partial w_{1}}{\partial x}(1, t)=0
\end{gathered}
$$

Equations (3.2) imply that

$$
u_{0}(x, t)=a(t), \quad w_{0}(x, t)=b(t),
$$

where $a(t)$ and $b(t)$ are, at this stage, arbitrary functions of $t$. In order to determine them we must proceed to the next step in the perturbation procedure.

Using (3.9), we can write the differential equations (3.3) and (3.4) as

$$
\begin{aligned}
& \frac{\partial^{2} u_{1}}{\partial x^{2}}=\frac{d a}{d t}-[\alpha(\lambda)+1] a+\beta(\lambda) b-f(\lambda, a, b) \equiv A(t), \\
& \frac{\partial^{2} w_{1}}{\partial x^{2}}=\frac{d b}{d t}-\beta(\lambda) a-[\alpha(\lambda)+1] b-g(\lambda, a, b) \equiv B(t) .
\end{aligned}
$$

Thus, $u_{1}(x, t)=\frac{1}{2} A(t) x^{2}+c_{1}(t) x+c_{2}(t)$ and $w_{1}(x, t)=\frac{1}{2} B(t) x^{2}+c_{3}(t) x+c_{4}(t)$, where the $c_{i}(t), i=1, \cdots, 4$, are arbitrary functions. The boundary conditions (3.5) and (3.6) imply that $c_{1}(t)=a(t)$ and $c_{3}(t)=b(t)$. Finally, to satisfy the boundary conditions (3.7) and (3.8), we find that we must have $A(t)+a(t)=0$ and $B(t)+b(t)$ 
$=0$; that is, we must have

$$
\begin{aligned}
& \frac{d a}{d t}=\alpha(\lambda) a-\beta(\lambda) b+f(\lambda, a, b), \\
& \frac{d b}{d t}=\beta(\lambda) a+\alpha(\lambda) b+g(\lambda, a, b) .
\end{aligned}
$$

Thus, the functions $a(t)$ and $b(t)$ are determined from compatibility conditions (3.12) and (3.13) which they must satisfy in order to generate a consistent perturbation procedure.

We now obtain the appropriate initial conditions for $a(t)$ and $b(t)$ by a standard matching procedure with the asymptotic form in the initial boundary layer. Let $\tilde{t}=t / \varepsilon$, and let $u(x, t)=u(x, \varepsilon \tilde{t}) \equiv U(x, \tilde{t}), w(x, t)=w(x, \varepsilon \tilde{t}) \equiv W(x, \tilde{t})$. With this change of variables we find that to first order in $\varepsilon$ the equations (1.9)-(1.16) become

$$
\begin{array}{ll}
U_{\tilde{t}}=U_{x x}, & W_{\tilde{t}}=W_{x x} \\
U_{x}(0, \tilde{t})=0, & W_{x}(0, \tilde{t})=0, \\
U_{x}(1, \tilde{t})=0, & W_{x}(1, \tilde{t})=0, \\
U(x, 0)=\phi(x), & W(x, 0)=\psi(x) .
\end{array}
$$

Therefore,

$$
\begin{gathered}
U(x, \tilde{t})=A_{0}+\sum_{n=1}^{\infty} A_{n} e^{-n^{2} \pi^{2} \tilde{t}} \cos n \pi x, \\
W(x, \tilde{t})=B_{0}+\sum_{n=1}^{\infty} B_{n} e^{-n^{2} \pi^{2} \tilde{t}} \cos n \pi x,
\end{gathered}
$$

where

$$
\begin{array}{ll}
A_{0}=\int_{0}^{1} \phi(\xi) d \xi, & A_{n}=2 \int_{0}^{1} \phi(\xi) \cos n \pi \xi d \xi \\
B_{0}=\int_{0}^{1} \psi(\xi) d \xi, & B_{n}=2 \int_{0}^{1} \psi(\xi) \cos n \pi \xi d \xi .
\end{array}
$$

Equations (3.15) and (3.16) give the first term (i.e., the zero order term) in the asymptotic expansion of the solution (the inner solution) of (1.9)-(1.16) in the boundary layer. The standard matching of inner and outer solutions now requires that

$$
\begin{aligned}
& \lim _{t \rightarrow 0}\left[u_{0}(x, t)\right]=\lim _{\tilde{t} \rightarrow \infty}[U(x, \tilde{t})], \\
& \lim _{t \rightarrow 0}\left[w_{0}(x, t)\right]=\lim _{\tilde{t} \rightarrow 0}[W(x, \tilde{t})] .
\end{aligned}
$$

Therefore, $a(0)=A_{0}$ and $b(0)=B_{0}$, and the equations governing $a(t)$ and $b(t)$ are

$$
\frac{d a}{d t}=\alpha(\lambda) a-\beta(\lambda) b+f(\lambda, a, b)
$$




$$
\begin{aligned}
& \frac{d b}{d t}=\beta(\lambda) a+\alpha(\lambda) b+g(\lambda, a, b), \\
& a(0)=A_{0}=\int_{0}^{1} \phi(\xi) d \xi \\
& b(0)=B_{0}=\int_{0}^{1} \psi(\xi) d \xi .
\end{aligned}
$$

The equations (3.14)-(3.18) show clearly that to first order in $\varepsilon$ the mechanism governing the solution of (1.9)-(1.16) is initially the standard linear diffusion process. After a time of the order $O(\varepsilon)$, the process, to first order in $\varepsilon$, is governed by the system (3.21)-(3.24); we shall now study this system.

4. Periodic solutions. The equations (3.21), (3.22) constitute a standard autonomous system normally treated by classical phase-plane techniques, and, in fact, much of what we shall establish here can be derived using a considerable amount of the well-established literature. However, we shall not follow such an approach for two reasons: (i) System (1.1)-(1.8) describes the simplest type of nonadiabatic tubular reactor. For large Peclet numbers (i.e., $P \gg 1$ ) our methods apply equally well to considerably more complicated problems in reactor theory [3], [11]. In these more difficult problems the equations analogous to (3.21), (3.22) which arise are not standard autonomous phase-plane systems, but much more difficult systems. Nevertheless, they can be handled by the techniques we shall develop here. (ii) The "two-timing" method which we shall use produces formulas for the solutions which are immediately interpretable physically and from which the stability of the solutions is also immediately resolved without recourse to further analysis. Thus, this method is simply much easier to use even on the standard phase-plane systems (and is applied equally well to the more difficult problems). When it works, the technique is spectacularly successful as has so often been the case where it has been applied in other problems (see [7] and [12] for examples).

The motivation for a two-timing approach as well as the proper scaling for the asymptotic analysis comes from the following reasoning: Consider, for example, the upper branch of the response diagram of Fig. 2 when we increase $D$ from slightly below $D_{P}$ to slightly above $D_{P}$ so that we pass through the point $P$ where the steady state changes from stable to unstable. As discussed in $\S 2$, we have concluded that the steady states to the right of point $P$ are unstable based upon a linearized perturbation theory which implies that perturbations from this steady state will initially grow exponentially in time. This (linearized) exponentially growing function cannot represent the solution for very long because clearly the nonlinear terms must then become important. If, in fact, this exponentially growing function tends to a stable oscillatory solution, as we conjectured in $\S 2$, then growth on another time scale must come into play so that in some sense the perturbation from the unstable steady state should exhibit a more or less typical multitime scale representation; namely, we expect a representation of the form $u(x, t)=A(\tau) P\left(t^{*}\right)$, where $P\left(t^{*}\right)$ represents a periodic oscillation on a so-called "fast time" $t^{*}$ and $A(\tau)$ represents "slow-time" modulation which perhaps 
approaches a constant value as time $t \rightarrow \infty$. Change of stability in a somewhat different type of heat conduction problem has also been studied in this way with a two-timing method by B. J. Matkowsky [13].

For ease of presentation here and in order not to obscure the basic method with lengthy algebraic calculations, we shall now perform our investigation on the system (3.21)-(3.24) for the special case that

$$
\begin{array}{ll}
f(\lambda, a, b)=-\lambda a^{3}, & g(\lambda, a, b)=-\mu \lambda a b^{2}, \\
\beta(\lambda)=\text { constant } \beta, & \alpha\left(\lambda_{0}\right)=\alpha^{\prime}\left(\lambda_{0}\right)=0, \quad \alpha^{\prime \prime}\left(\lambda_{0}\right) \neq 0 .
\end{array}
$$

In fact, such a choice is an excellent model for a simple first order reaction for the system (1.1)-(1.8), where the $\beta$ here corresponds to the $\kappa$ in (1.1) and $\mu$ corresponds to the transformed $B$ of $(1.1)$; see [4]. We would like to point out that our entire analysis can be carried out for the general system (1.9)-(1.16) under conditions $\mathrm{H}-1$ to $\mathrm{H}-4$ as well as for the general reactor problems involving higher order chemical kinetics for the rate functions $f$ and $g$. This general treatment necessarily requires considerably more complicated algebraic manipulation, and, in fact, for the general reactor kinetics, some of the algebraic equations required the numerical specification of certain physical contents and numerical procedures for solving involved algebraic expressions. Such specific information would be of use only in specific chemical processes, but, in fact, for certain interesting commonly occurring chemical constants, A. B. Poore [5] has carried out these calculations.

The only necessary tool which we shall need in carrying out the two-timing formalism is an elementary fact from ordinary differential equations which we shall state in the form of an easily referenced lemma.

LEMMA. The general solution of

$$
\begin{aligned}
& \frac{d x}{d t}+y=m \sin t+n \cos t, \\
& \frac{d y}{d t}-x=p \sin t+q \cos t
\end{aligned}
$$

is

$$
\begin{aligned}
& x(t)=A \sin t+B \cos t+\left(\frac{m-q}{2}\right) t \sin t+\left(\frac{n+p}{2}\right) t \cos t+\left(\frac{n-p}{2}\right) \sin t \\
& y(t)=-A \cos t+B \sin t+\left(\frac{n+p}{2}\right) t \sin t-\left(\frac{m-q}{2}\right) t \cos t+\left(\frac{m+q}{2}\right) \sin t
\end{aligned}
$$

Thus, in order to suppress secular terms (i.e., in order to have solutions bounded for all $t \geqq 0$ ) it is sufficient to require $m-q=0$ and $n+p=0$.

We define $\delta$ by the relationship $\delta=\lambda-\lambda_{0}$, and we assume that

$$
\begin{aligned}
& a \equiv a\left(t^{*}, \tau\right)=\delta a_{1}\left(t^{*}, \tau\right)+\delta^{2} a_{2}\left(t^{*}, \tau\right)+\cdots \\
& b \equiv b\left(t^{*}, \tau\right)=\delta b_{1}\left(t^{*}, \tau\right)+\delta^{2} b_{2}\left(t^{*}, \tau\right)+\cdots
\end{aligned}
$$


where the "slow time" $\tau$ and "fast time" $t *$ are defined by

$$
\begin{gathered}
\tau=\delta^{2} t \\
t^{*}=\left(1+\delta \omega_{1}+\delta^{2} \omega_{2}+\cdots\right) t .
\end{gathered}
$$

Much of the technique has already been employed with judicious choice of the forms (4.2)-(4.5). The further manipulation (see Cole [7] or Kevorkian [12] for its exposition) requires that the $\omega_{i}$ and the other unknowns which will occur shall be chosen according to the principle that we suppress secular terms in such a way that we generate a self-consistent procedure for determining bounded functions $a_{i}\left(t^{*}, \tau\right)$ and $b_{i}\left(t^{*}, \tau\right)$ with modulation only on the slow time scale $\tau$. We shall now carry out this procedure.

With the definitions (4.4) and (4.5) we find that

$$
\frac{d}{d t}=\left(1+\delta \omega_{1}+\delta^{2} \omega_{2}+\cdots\right) \frac{\partial}{\partial t}+\delta^{2} \frac{\partial}{\partial \tau} .
$$

Thus, substituting (4.2)-(4.5) into (3.21), (3.22) under the requirements (4.1) and equating the coefficients of like powers of $\delta$, we obtain

$$
\begin{gathered}
\frac{\partial a_{1}}{\partial t^{*}}=-\beta b_{1}, \\
\frac{\partial b_{1}}{\partial t^{*}}=\beta a_{1}, \\
\frac{\partial a_{2}}{\partial t^{*}}+\beta b_{2}=-\omega_{1} \frac{\partial a_{1}}{\partial t^{*}} \\
\frac{\partial b_{2}}{\partial t^{*}}-\beta a_{2}=-\omega_{1} \frac{\partial b_{1}}{\partial t^{*}}, \\
\frac{\partial a_{3}}{\partial t^{*}}+\beta b_{3}=-\frac{\partial a_{1}}{\partial \tau}-\omega_{1} \frac{\partial a_{2}}{\partial t^{*}}-\omega_{2} \frac{\partial a_{1}}{\partial t^{*}}+\frac{\alpha^{\prime \prime}\left(\lambda_{0}\right)}{2} a_{1}-\lambda_{0} a_{1}^{3}, \\
\frac{\partial b_{3}}{\partial t^{*}}-\beta a_{3}=-\frac{\partial b_{1}}{\partial \tau}-\omega_{1} \frac{\partial b_{2}}{\partial t^{*}}-\omega_{2} \frac{\partial b_{1}}{\partial t^{*}}+\frac{\alpha^{\prime \prime}\left(\lambda_{0}\right)}{2} b_{1}-\mu \lambda_{0} a_{1} b_{1}^{2} .
\end{gathered}
$$

The solution of (4.6) is given by

$$
\begin{aligned}
& a_{1}\left(t^{*}, \tau\right)=A(\tau) \sin \beta t^{*}+B(\tau) \cos \beta t^{*}, \\
& b_{1}\left(t^{*}, \tau\right)=-A(\tau) \cos \beta t^{*}+B(\tau) \sin \beta t^{*},
\end{aligned}
$$

where the unknown functions $A(\tau)$ and $B(\tau)$ will be determined at a later stage of the perturbation procedure. With these formulas for $a_{1}\left(t^{*}, \tau\right)$ and $b_{1}\left(t^{*}, \tau\right)$ used on the right-hand side of (4.7), we find that (4.7) becomes

$$
\begin{aligned}
& \frac{\partial a_{2}}{\partial t^{*}}+\beta b_{2}=-\omega_{1} \beta A(\tau) \cos \beta t^{*}+\omega_{1} \beta B(\tau) \sin \beta t^{*} \\
& \frac{\partial b_{2}}{\partial t^{*}}-\beta a_{2}=\omega_{1} \beta A(\tau) \sin \beta t^{*}-\omega_{1} \beta B(\tau) \cos \beta t^{*}
\end{aligned}
$$


Using our lemma to suppress secular terms, we see immediately that we must require that $\omega_{1}=0$. Thus,

$$
\begin{aligned}
& a_{2}\left(t^{*}, \tau\right)=C(\tau) \sin \beta t^{*}+D(\tau) \cos \beta t^{*}, \\
& b_{2}\left(t^{*}, \tau\right)=-C(\tau) \cos \beta t^{*}+D(\tau) \sin \beta t^{*},
\end{aligned}
$$

where the $C(\tau)$ and $D(\tau)$ are to be determined at a later stage in our perturbation scheme. The system (4.8) now becomes

$$
\begin{aligned}
\frac{\partial a_{3}}{\partial t^{*}}+\beta b_{3}= & -\frac{d A}{d \tau} \sin \beta t^{*}-\frac{d B}{d \tau} \cos \beta t^{*}-\omega_{2} \beta A \cos \beta t^{*}+\omega_{2} \beta B \sin \beta t^{*} \\
& +\frac{\alpha^{\prime \prime}\left(\lambda_{0}\right)}{2} A \sin \beta t^{*}+\frac{\alpha^{\prime \prime}\left(\lambda_{0}\right)}{2} B \cos \beta t^{*}-\lambda_{0} A^{3} \sin ^{3} \beta t^{*} \\
& -3 \lambda_{0} A^{2} B \sin ^{2} \beta t^{*} \cos \beta t^{*}-3 \lambda_{0} A B^{2} \sin \beta t^{*} \cos ^{2} \beta t^{*}-\lambda_{0} B^{3} \cos ^{3} \\
& -\lambda_{0} B^{3} \cos ^{3} \beta t^{*}
\end{aligned}
$$

$$
\begin{aligned}
\frac{\partial b_{3}}{\partial t^{*}}-\beta a_{3}= & \frac{d A}{d \tau} \cos \beta t^{*}-\frac{d B}{d \tau} \sin \beta t^{*}-\omega_{2} \beta A \sin \beta t^{*}-\omega_{2} \beta B \cos \beta t^{*} \\
& -\frac{\alpha^{\prime \prime}\left(\lambda_{0}\right)}{2} A \cos \beta t^{*}+\frac{\alpha^{\prime \prime}\left(\lambda_{0}\right)}{2} B \sin \beta t^{*}-\mu \lambda_{0} A B^{2} \sin ^{3} \beta t^{*} \\
& -\mu \lambda_{0}\left(B^{3}-2 A^{2} B\right) \sin ^{2} \beta t^{*} \cos \beta t^{*} \\
& -\mu \lambda_{0}\left(A^{3}-2 A B^{2}\right) \sin \beta t^{*} \cos \beta t^{*}-\mu \lambda_{0} A^{2} B \cos ^{3} \beta t^{*}
\end{aligned}
$$

By employing certain well-known trigonometric identities (or, equivalently, by developing the right-hand sides in a Fourier series), we can write (4.12) as

$$
\begin{aligned}
\frac{\partial a_{3}}{\partial t^{*}}+\beta b_{3}= & {\left[-\frac{d A}{d \tau}+\omega_{2} \beta B+\frac{\alpha^{\prime \prime}\left(\lambda_{0}\right)}{2} A-\frac{3}{4} \lambda_{0} A^{3}-\frac{3}{4} \lambda_{0} A B^{2}\right] \sin \beta t^{*} } \\
& +\left[-\frac{d A}{d \tau}-\omega_{2} \beta A+\frac{\alpha^{\prime \prime}\left(\lambda_{0}\right)}{2} B-\frac{3}{4} \lambda_{0} A^{2} B-\frac{3}{4} \lambda_{0} B^{3}\right] \cos \beta t^{*} \\
& + \text { (higher harmonics) }
\end{aligned}
$$

$$
\begin{aligned}
\frac{\partial b_{3}}{\partial t^{*}}-\beta a_{3}= & {\left[-\frac{d B}{d \tau}-\omega_{2} \beta A+\frac{\alpha^{\prime \prime}\left(\lambda_{0}\right)}{2} B-\frac{3}{4} \mu \lambda_{0} A B^{2}\right.} \\
& \left.-\frac{1}{4} \mu \lambda_{0}\left(A^{3}-2 A B^{2}\right)\right] \sin \beta t^{*}+\left[\frac{d A}{d \tau}-\omega_{2} \beta B\right. \\
& \left.-\frac{\alpha^{\prime \prime}\left(\lambda_{0}\right)}{2} A-\frac{1}{4} \mu \lambda_{0}\left(B^{3}-2 A^{2} B\right)-\frac{3}{4} \mu \lambda_{0} A^{2} B\right] \cos \beta t^{*} \\
& + \text { (higher harmonics). }
\end{aligned}
$$


Now, suppression of secular terms according to our lemma implies that we require $-2 \frac{d A}{d \tau}+2 \omega_{2} \beta B+\alpha^{\prime \prime} A-\frac{3}{4} \lambda A^{3}-\frac{3}{4} \lambda_{0} A B^{2}+\frac{1}{4} \mu \lambda_{0}\left(B^{3}-2 A^{2} B\right)+\frac{3}{4} \mu \lambda_{0} A^{2} B=0$,

$$
\begin{aligned}
& -2 \frac{d B}{d \tau}-2 \omega_{2} \beta A+\alpha^{\prime \prime} B-\frac{3}{4} \lambda_{0} A^{2} B-\frac{3}{4} \lambda_{0} B^{3}-\frac{3}{4} \mu \lambda_{0} A B^{2}-\frac{1}{4} \mu \lambda_{0}\left(A^{3}-2 A B^{2}\right) \\
& \quad=0
\end{aligned}
$$

or, equivalently,

$$
-2 \frac{d A}{d \tau}+2 \omega_{2} \beta B+\alpha^{\prime \prime}\left(\lambda_{0}\right) A-\frac{3}{4} \lambda_{0} A\left(A^{2}+B^{2}\right)+\frac{1}{4} \mu \lambda_{0} B\left(A^{2}+B^{2}\right)=0,
$$

$$
-2 \frac{d B}{d \tau}-2 \omega_{2} \beta A+\alpha^{\prime \prime}\left(\lambda_{0}\right) B-\frac{3}{4} \lambda_{0} B\left(A^{2}+B^{2}\right)-\frac{1}{4} \mu \lambda_{0} A\left(A^{2}+B^{2}\right)=0 .
$$

The periodic nature of the solutions of (3.21), (3.22) for small $\delta>0$ can now be established by employing a device due to J. D. Cole [7]. Multiply the first of equations (4.15) by $A$ and the second by $B$, and then add the equations to obtain

$$
-\frac{d R}{d \tau}+\alpha^{\prime \prime}\left(\lambda_{0}\right) R-\frac{3}{4} \lambda_{0} R^{2}=0
$$

where

$$
R=A^{2}+B^{2} .
$$

The solution of (4.16) is easily found to be

$$
R(\tau)=\frac{4 \alpha^{\prime \prime}\left(\lambda_{0}\right)}{3 \lambda_{0}} \frac{1}{1+k e^{-\alpha^{\prime \prime}\left(\lambda_{0}\right) \tau}}, \quad k=\text { const } .
$$

Thus, equations (4.2), (4.3), (4.17), (4.18) clearly show an approach to a limit cycle of amplitude

$$
2\left[\frac{\alpha^{\prime \prime}\left(\lambda_{0}\right)}{3 \lambda_{0}}\right]^{1 / 2} \delta
$$

Thus, as claimed in $\S 2$ we have established that periodic solution branches of the form (4.19) to $O(\delta)$ bifurcate from the points $R$ and $S$ of Fig. 1 and from the points $P$ and $Q$ of Fig. 2. Note that the stability of these oscillatory branches follows immediately by writing (4.16) in the form

$$
\frac{d R}{d \tau}=\frac{3 \lambda_{0} R}{4}\left[\frac{4 \alpha^{\prime \prime}\left(\lambda_{0}\right)}{3 \lambda_{0}}-R\right]
$$

from which we can immediately conclude that for $R(\tau)$ less (greater) than its steady state value $4 \alpha^{\prime \prime}\left(\lambda_{0}\right) / 3 \lambda_{0}$ we have $d R / d \tau$ greater (less) than zero, implying motion towards the steady state (i.e., stability). 
5. Multiple stable and unstable periodic solutions. For values of the physical parameters $\kappa, B, P, T_{c}, \gamma, h, l$ in certain ranges (see [5]) the situation illustrated in Fig. 2 remains the same with regard to steady states and their stability, but the bifurcation of periodic solutions is very different from that illustrated in Fig. 4. The situation which does occur is that illustrated in Fig. 5, and we shall now discuss this case.

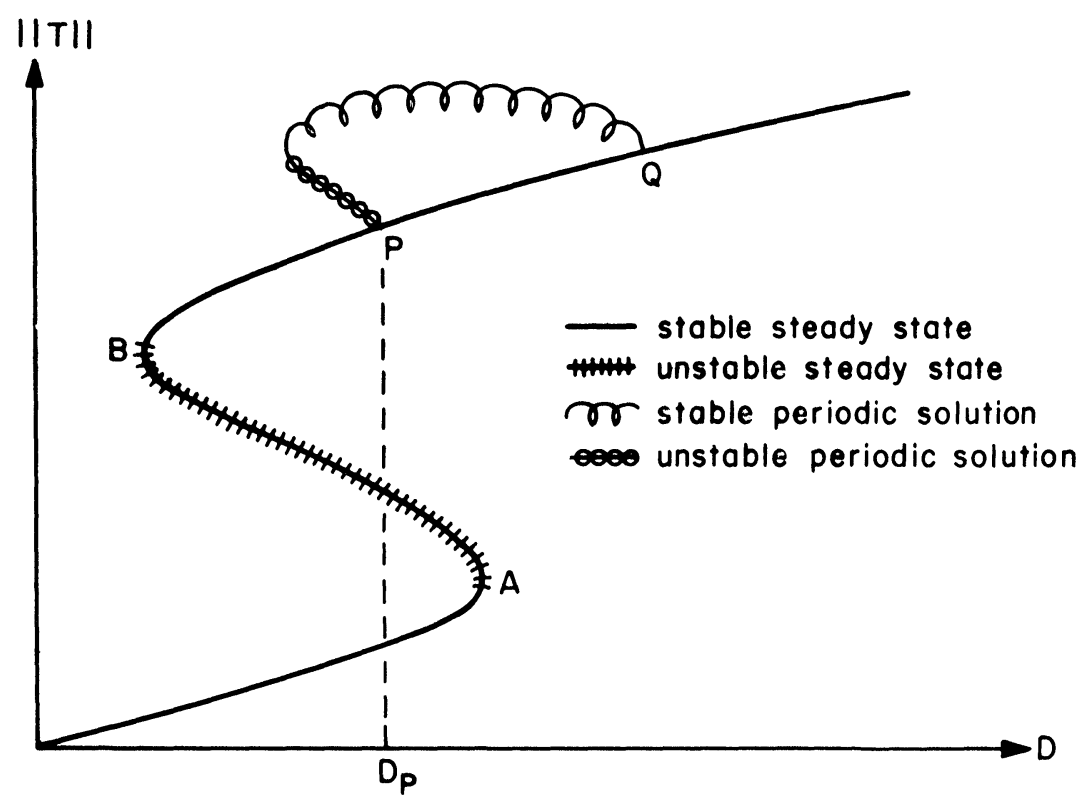

FIG. 5

When physical situations more complicated than those modeled by equations (4.1) are considered, it can happen that in the modeling or in the actual equations we obtain $\alpha^{\prime}\left(\lambda_{0}\right) \neq 0$ together with nonlinearities such that the point $P$ of Fig. 2 or Fig. 5 becomes a bifurcation point from which an unstable branch of periodic solutions bifurcates. This situation is presently being studied. Preliminary work of Cohen and Poore indicates the following: The "two-timing" procedure of the preceding section is no longer applicable as it stands (because it will not apply to the determination of an unstable limit cycle). However, suitable modifications and other techniques indicate that the situation is that indicated in Fig. 5. That is, an unstable branch of periodic solutions bifurcates to the left at point $P$. Furthermore, a stable periodic solution (a limit cycle of the equations analogous to (3.21), (3.22)) surrounds the unstable one. A stable branch of periodic solutions bifurcates to the left at point $Q$. We conclude that the periodic branch from $P$ to $Q$ is as shown in Fig. 5. For a continuous stirred tank reactor, Hlavacek, Kubicek and Jelinek [4] have given numerical calculations of the solutions for values of $D$ slightly below $D_{P}$. They find the stable limit cycle which exists surrounding the stable steady state, but their procedures do not indicate an unstable solution. Our results explain this situation and apply also to tubular reactors; this work will appear in [14]. 


\section{REFERENCES}

[1] D. K. Winegardner AND R. A. Schmitz, Dynamics of heterogeneous reaction at a stagnation point, AIAA J., 5 (1967), pp. 1589-1595.

[2] R. C. LindBuRg AND R. A. Schmitz, Dynamics of heterogeneous reaction at a stagnation point: numerical study of nonlinear transient effects, Internat. J. Heat Mass Transfer, 14 (1971), pp. 718-721.

[3] V. Hlavacek and H. Hofmann, Modeling of chemical reactors-XIX. Transient axial heat and mass transfer in tubular reactors. The stability considerations-I, Chem. Engrg. Sci., 25 (1970), pp. $517-1526$.

[4] V. HlavaceK, M. Kubicek And J. Jelinek, Modeling of chemical reactors-XVII. Stability and oscillatory behaviour of the CSTR, Ibid., 25 (1970), pp. 1441-1461.

[5] A. B. POORE, Stability and bifurcation phenomena in chemical reactor theory, Ph.D. thesis, California Institute of Technology, Pasadena, 1972.

[6] - Multiplicity, stability, and bifurcation of periodic solutions in problems from chemical reactor theory, to appear.

[7] J. D. Cole, Perturbation Methods in Applied Mathematics, Blaisdell, Waltham, Mass., 1968.

[8] D. S. CoHEN, Multiple stable solutions of nonlinear boundary value problems arising in chemical reactor theory, this Journal, 20 (1971), pp. 1-13.

[9] - Multiple solutions of singular perturbation problems, SIAM J. Math. Anal., 3 (1972), pp. 72-82.

[10] V. Hlavacek, H. Hofmann and M. KubiceK, Modeling of chemical reactors-XXIV. Transient axial heat and mass transfer in tubular reactors. The stability considerations-II, Chem. Engrg. Sci., 25 (1971), pp. 1629-1634.

[11] C. R. MCGOWIn AND D. D. PeRLMUTTER, Tubular reactor steady state and stability characteristics, AIChE J., 17 (1971), pp. 831-849.

[12] J. Kevorkian, The two variable expansion procedure for the approximate solution of certain nonlinear differential equations, Lectures in Appl. Math., vol. 7, Space Math., Part III, American Mathematical Society, Providence, 1966. Also in Proc. Yale Univ. Summer Institute on Dynamical Astronomy.

[13] B. J. Matkowsky, A simple nonlinear dynamic stability problem, Bull. Amer. Math. Soc., 76 (1970), pp. 620-625.

[14] D. S. COHEN AND A. B. PoOre, Tubular chemical reactors: The "lumping approximation" and bifurcation of oscillatory states, to appear. 